\title{
ACUTE SUPPURATIVE THYROIDITIS CAUSED BY BURKHOLDERIA CEPACIA
}

Ilhan TARKUN ${ }^{1}$, Ozlem Zeynep AKYAY ${ }^{1}$, Berrin CETINARSLAN ${ }^{1}$, Zeynep CANTURK ${ }^{1}$, Alev SELEK ${ }^{1}$ Fatma $^{2}$ Tugba CATAN ${ }^{2}$,

${ }^{1}$ Section of Endocrinology, Department of Internal Medicine, Kocaeli University, Kocaeli, Turkey

'Department of InternalMedicine, Kocaeli University, Kocaeli, Turkey

\section{OBJECTIVES}

The thyroid is generally resistant to infections due to its encapsulated location, high iodine concentration, hydrogen peroxide production, and high levels of blood and lymphoid circulation therefore acute suppurative thyroiditis is rarely seen. The most common agents are Staphilococcus and Streptococcus. In this case we present acute suppurative

thyroiditis caused by Burkholdeiracepacia,that is a rare infectious agent in adults.
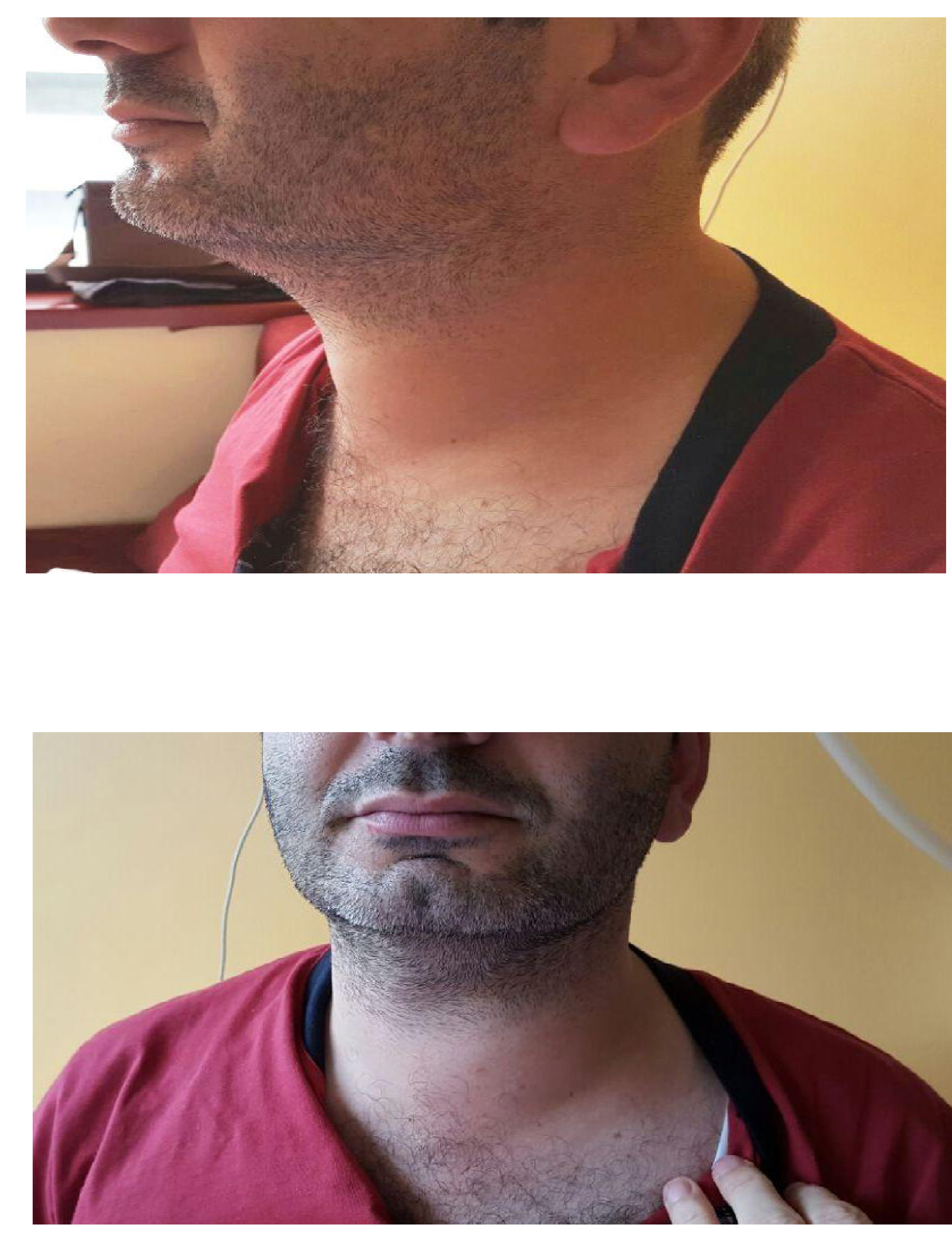

CASE

34 year-old-male, admitted to our clinic with sudden onsetleft- sided neck swelling with pain and redness. There was no history of chronic disease, recently upper respiratory tract infection or trauma. A painfull nodule was palpated on the left lob of his thyroid gland. There were monocytosis $1.011 \times 103 \mu \mathrm{l}(0.00-0.900 \times 103)$, increased sedimentation rate; $52 \mathrm{~mm} / \mathrm{h}(<20 \mathrm{~mm} / \mathrm{h})$ and C- reactive protein (CRP) level; 14.2 $\mathrm{mg} / \mathrm{dl}(0-0.5)$. Thyroid hormoneswere in normal limits. Neck ultrasonography revealed $6 \times 5 \mathrm{~mm}$ high density cystic complex nodule, increased vascularityof left lobe and reactive lymph nodes localized at left jugular are. Basedon the clinical and laboratory findings, acute suppurative thyroiditis was decided.Fine needle aspiration from the cystic nodule and blood culture was performed for the microbiological identification. Amoksicilin-clavulonic acid treatment was started. Ciprofloxacin and amikacin sensitiveBurkholdeiraCepacia identified on the cystic nodule aspiration at the seventh day of the treatment. Antibiotic treatment switched to the ciprofloxacin. All the symptoms of the disease were regressed, sedimentation and CRP rates normalized at the twentiethday of the ciprofloksasin treatment. There was not any recurrence

\section{CONCLUSIONS}

Acute suppurative thyroiditis is a rare infection disease. When the clinician encountered with acute suppurative thyroiditis, abscess aspiration culture and the blood culture for the microbiological identification and antibiogram should be performed.Because rare microorganism would be causative agent of existing acute suppurative thyroiditis. 